\title{
¿Hasta dónde influyen las agendas internacionales en los sistemas educativos centroamericanos? Un marco teórico para su análisis
}

\section{$¿$ How much influence does the international agenda have over the Central American educational systems?}

\section{A theoretical framework}

\author{
Mónica E. Salazar Vides*1 \\ elizabethsalazarvides@gmail.com
}

\section{Resumen}

En este ensayo se propone un marco teórico para el estudio del influjo internacional sobre los procesos de reforma educativa en Centroamérica iniciados en los años noventa, a partir de las particularidades políticas y culturales de los contextos centroamericanos. Se hizo el ejercicio de poner en diálogo la corriente «internacional» $\mathrm{y}$ «cultural» de la economía política crítica de la educación para comprender la influencia de los organismos internacionales y sus agendas educativas en los procesos de reforma educativa,

* Investigadora, Instituto de investigación y proyección sobre dinámicas globales y territoriales de la Universidad Rafael Landívar, Guatemala.

${ }^{1}$ Este ensayo se extrae del marco teórico generado para la investigación en marcha: "Configuración de los modelos de la calidad educativa en Centroamérica: reformas educativas, transiciones democráticas y ajustes económicos», en el marco de la Red de Estudios sobre Educación en Centroamérica (REDÉCA), integrada por Universidad Rafael Landívar de Guatemala, Universidad Centroamericana «José Simeón Cañas» de El Salvador, Universidad Centroamericana de Nicaragua, Universidad Pedagógica «Francisco Morazán» de Honduras, Fe y Alegría Honduras y, Fe y Alegría Centroamérica.

Recibido 11 de septiembre de 2018 / Aceptado 26 de octubre de 2018 DOI: https://dx.doi.org/10.5377/paradigma.v25i40.7532

$<$ Paradigma> Revista de Investigación Educativa 40 Noviembre 2018/ ISSN: 1817-4221

Los articulos de la Revista Electrónica Paradigma del Instituto de Investigación y Evaluación Educativas y Sociales de la Universidad Pedagógica Nacional Francisco Morazán, de Honduras, se comparten bajo términos de la Licencia Creative Commons: Se permite que otros puedan descargar las obras y compartirlas con otras personas, siempre y cuando se 
en función de las dinámicas históricas nacionales. En otras palabras, para entender por una parte, la interacción entre la globalización como capitalismo global, y los sistemas educativos nacionales centroamericanos como parte de los Estados-Nación; y por otra, las relaciones de poder entre los actores nacionales y su capacidad de agencia para hegemonizar ideológicamente a través del proceso de reforma educativa, una visión de sociedad y ciudadano en tanto clase, etnia, religión y género en un país.

Palabras clave: reformas educativas en Centroamérica, sociología de la educación, economía política, globalización, hegemonía ideológica

\begin{abstract}
This essay proposes a theoretical framework to analyze the international input on Central American educational reform processes in the early 90s, in light of the political and cultural background in the region. The framework is based on a dialogue between the critical international and cultural perspectives of political economy applied in studies of educational sociology, in order to understand the influence that international agencies and their respective educational agendas have on educational reform processes in each national historical setting. The essay sustains that this theoretical analysis helps to understand how globalization, mainly understood as global capitalism, interacts with the national educational systems as a part of the nation state; and also, the power relations between different national agents, and their capacity to produce, thru educational reform process, an ideological hegemonic vision of society and ideal citizenship according to a country's ideas of class, ethnicity, gender and religion.
\end{abstract}


¿Hasta dónde influyen las agendas internacionales en los sistemas

educativos centroamericanos?

Un marco teórico para su análisis

Keywords: educational reforms in Central America, educational sociology, political economy, globalization, ideological hegemony

\section{Economía política crítica de la educación}

Mucho se ha escrito sobre la influencia de los organismos internacionales y sus agendas educativas en los sistemas educativos nacionales de países del llamado «Tercer Mundo» (Edwards, 2018; Dale, 1999; Verger, Fontdevila \& Zancajo, 2016). En este ensayo no se pretende argumentar en contra de dicha influencia, pero sí generar un marco teórico para entender la influencia de las dinámicas políticas y culturales nacionales en la adopción o rechazo de las agendas educativas internacionales en los Procesos de Reforma Educativa -PRE- en Centroamérica. ${ }^{2} Y$ es que si bien es innegable que organismos internacionales como el Banco Mundial han condicionado las reformas educativas iniciadas en los años noventa en Centroamérica (Edwards, 2018), vale la pena comprender cómo y porqué los actores nacionales se posicionaron a favor o en contra de las agendas internacionales. Argumentamos que esta congruencia o conflicto de intereses internacionales y nacionales puede comprenderse a profundidad si se estudian las luchas político-ideológicas y los procesos histórico-culturales de cada país centroamericano.

Desarrollamos un marco teórico fundamentado en la «economía política crítica»-EPC-. Escogimos la EPC pues de acuerdo a Edwards (2018), citando a Novelli (2014), se caracteriza por: a) considerar el contexto histórico e institucional de los fenómenos bajo estudio; b) examinar los grupos sociales

\footnotetext{
${ }^{2}$ Cabe aclarar que este ensayo brinda herramientas teóricas para analizar la influencia de las agendas internacionales y de los contextos nacionales en los procesos de reforma educativa en Centroamérica, más no contiene un análisis en sí de dichos contextos o influencias. Se proponen más bien, medios para analizar dichos fenómenos 
involucrados, y sus intereses de clase; c) buscar de-construir conceptos, a través de la relación entre argumento, ideología e intereses de clase de los grupos involucrados; d) prestar atención a la distribución y reproducción desigual de recursos, del poder y del conocimiento, que tiende a vincularse a jerarquías sociales más amplias; y d) no se enfoca en «cosas dadas», sino que en procesos: cómo lo que se llegó a producirse. Ahora bien, para el estudio de los PRE seleccionamos dos vertientes de la EPC: la economía política crítica internacional-EPCI-y la economía política crítica cultural-EPCC-.

La EPCInos ayudará a estudiar el influjo de las agendas educativas globales de los organismos internacionales sobrelos PRE de países que, como los centroamericanos, son considerados «Tercermundistas». Tomaremosel desarrollo teóricode Edwards (2018) como referencia. Su objeto deestudio constituye lo que élllama «Procesos Internacionales deFormación dePolíticas Educativas», es decir, «la manera en que las influencias internacionales afectan los procesos oficialmente nacionales que se traducen en políticas educativas» (2014, p. 3). El análisis de la influencia de los organismos internacionales sobre PRE desarrollados por sistemas educativos nacionales conduce en términos más abstractos a la tensión entre globalización y autonomía de los Estados-Nación. Consideramos en consecuencia, que la EPCI nos será especialmente útil para comprender la interacción entre los niveles internacional y nacional en los PRE.

Michael Apple(1993 y 1993b), por otra parte, es nuestro principal referente dentro la EPCC. Apple intenta traspasar el determinismo económico sobre todos los aspectos dela vida social, incluyendoel sistema educativo, a través delestudiodela cultura y la ideología. El papel del neoconservadurismo (cultura) es igual de importante queel proyectoneoliberal (economía) para comprender los PRE. Apple disiente en consecuencia, delos estudios de Bowles y Ginstis, y deAlthusser sobre 
¿Hasta dónde influyen las agendas internacionales en los sistemas

educativos centroamericanos?

Un marco teórico para su análisis

la «correspondencia» entre el sistema económico capitalista, y los aparatos y contenidos educativos. Más bien visualiza una «conexión» entre dos esferas interrelacionadas einterdependientes: educación (cultura) y economía, que puede estudiarse a través de las pugnas político-ideológicas entre diferentes sectores por hegemonizar una visión de sociedad y ciudadano a través del sistema educativo. La EPCC nos ayudará a comprender cómo influyen las luchas de poder entre los diversos actores y los factores culturales eideológicos nacionales en los PRE.

A través del dialogo entre la EPCI y EPCC intentaremos resolver, para el caso de los estudios de los PRE, el clásico problema entre «estructura» $\mathrm{y}$ «agencia». Puesto que la EPCI aporta más luces para comprender la dimensión estructural en la que los agentes participantes de los PRE se encuentran inmersos, yendo de los condicionantes globales (capitalismo global) a los nacionales (aparato educativo nacional).Mientras que la EPCC puedeayudarnos a profundizar más en el ámbito nacional-la globalización no aparece como categoría de análisis en Apple-y el papel de la ideología y la cultura en las luchas de poder entre actores nacionales que en tanto agentes, intentaron hegemonizar $s u$ visión sobrela educación en los PRE. Desarrollaremosa continuación estas dos dimensiones, es decir, a) la economía política crítica internacional y b) la economía política crítica cultural.

\section{Economía política crítica internacional: dinámicas globales y condicionantes estructurales.}

Con el advenimiento del neoliberalismo y la globalización difícilmente se pueden analizar procesos educativos como dinámicas nacionales cerradas (Tarabini y Bonal, 2011). Esto nos obliga a reflexionar sobre el concepto de la «globalización» en relación a las agendas educativas internacionales.

Los articulos de la Revista Electrónica Paradigma del Instituto de Investigación y Evaluación Educativas y Sociales de la Universidad Pedagógica Nacional Francisco Morazán, de Honduras, se comparten bajo térrminos de la Licencia Creative Commons: Se permite que otros puedan descargar las cbras y compartirlas con otras personas, siempre y
reconozca su autoria, pero no se pueden cambiar de ninguna manera ni se pueden utilizar comercialmente. 
Globalización y agendas educativas internacionales.

De acuerdo a Nederveen (2009), se tiende a representar la «globalización» como un proceso de homogenización cultural que se da bajo los estándares de Occidente. La globalización así entendida explica Massey (2005), se rige bajo las pautas ideológicas de la «modernidad». Si trasponemos esta noción dominante de la globalización al fenómeno de las agendas educativas mundiales encontraremos un enfoque coincidente y otro, disidente. Según Roger Dale (2000), existen dos teorías sobre la relación entre la globalización y las agendas educativas mundiales. La primera es la «Cultura Educativa Mundial»-CEM-, propuesta por John Meyer y colegas de la Universidad de Stanford; y la segunda, la «Agenda Educativa Globalmente Estructurada» AEGE- propuesta por Dale.

El presupuesto central de la CEM es que la homogenización de las categorías curriculares a nivel mundial puede explicarse a través de modelos universales sobre educación, Estado y sociedad, y no por factores nacionales. Meyer y colegas consideran que los Estados organizan la educación nacional, incrustados en una sociedad global, y orientados por una ideología dominante mundial que exalta los valores occidentales de la «modernidad». Según Meyer y colegas (1987, citado por Dale, 2000), valores occidentales tales como la racionalidad, el progreso, el individualismo y la justicia se encuentran diseminados por modelos educativos y curriculares cada vez más estandarizados, los cuales son consecuencia natural y positiva de la penetración de la vida moderna y su matriz cultural en los territorios del globo. Esta sería la versión de las agendas educativas mundiales que coincide con la idea de la globalización como un movimiento inevitable y positivo hacia la homogenización cultural. 
¿Hasta dónde influyen las agendas internacionales en los sistemas

educativos centroamericanos?

Un marco teórico para su análisis

Dale (2000), basándose en la EPCI, propone en contraposición, la existencia de una AEGE. En ésta se concibe a la economía capitalista mundial como la fuerza dinamizadora de la globalización, que como tal, intenta esparcir sus intereses a los sistemas educativos nacionales. Lo que el mismo autor argumenta, es que los Estados están cediendo voluntariamente aspectos significativos de su soberanía a organizaciones internacionales por la necesidad de insertarse a una economía global de mercado. La educación en este caso funge como un factor medular para la competitividad de los Estados a través de la formación de recursos humanos o capital humano mejor capacitado. La estandarización educativa bajo estos términos, lejos de reflejar la adopción natural y progresiva de valores modernos universales y neutrales, constituiría una muestra de la necesidad de instalar valores afines a la acumulación del capital en el globo y naturalizar su diseminación. Esta versión de las agendas educativas globales disiente de la concepción dela globalización como estandarización cultural o modernidad, concibiéndola más bien como capitalismo global. ${ }^{3}$

Si tomamos como punto de partida las reformas educativas centroamericanas cuyos inicios se remontan a finales de los años ochenta y principios de los años noventa, vemos que coinciden en el tiempo con procesos económicos de ajuste estructural de segunda generación en cada país. Ajustes estructurales que se rigen por la ideología neoliberal del Consenso de Washington(Dale, 2000). Por ende, la perspectiva de la AEGE es más útil para comprender de manera crítica la relación entre las agendas educativas internacionales y la

\footnotetext{
${ }^{3}$ Las teorías del capitalismo global, según Robinson (2007), tienden a concebir la «globalización capitalista», como la fase actual del capitalismo mundial.

Los articulos de la Revista Electrónica Paradigma del Instituto de Investigación y Evaluación Educativas y Sociales de la Universidad Pedagógica Nacional Francisco Morazán, de Honduras, se comparten bajo términos de la Licencia Creative Commons: Se permite que otros puedan descargar las obras y compartirlas con otras personas, siempre y
reconozca su autoria, pero no se pueden cambiar de ninguna manera ni se pueden utilizar comercialmente.
} 
globalización entendida como capitalismo global en Centroamérica. No obstante, su principal riesgo reside en considerar que los procesos educativos nacionales se explican por la imposición de agendas educativas internacionales determinadas por el capitalismo global, sin analizar el rol de los Estados y las luchas políticas entre los actores nacionales en la agenda educativa de un país en específico. En otras palabras, los procesos económicos globales no han hecho de los Estados-Nación instituciones irrelevantes u obsoletas (Dale, 2000).

Capitalismo global, y sistema educativo nacional como aparato del Estado.

De acuerdo a Tarabini y Bonal, la globalización «tiene efectos que modifican las bases de intervención del Estado en educación, pero este sigue preservando, en la mayoría de países, su capacidad de provisión, regulación y financiación de la educación» (2011, p. 238). O como explica Bonal, la educación no está sujeta a dinámicas económicas únicamente-reproducción de la división social del trabajo, de la ideología del consumo etc.- sino que se encuentra atravesada por «relaciones de poder entre los grupos en el propio aparato político, los problemas de financiación del Estado y los procesos de desregulación, o el coste político de un sistema educativo» (1998, p. 181). Por tanto, lo que debemos analizar, expresa Dale (2000) es cómo el capitalismo global ha influido sobre la naturaleza de los Estados Nación y por ende, en políticas sociales como las educativas.

Los Estados para acomodarse a estas nuevas condiciones económicas mundiales han adoptado, según Cerny (1997), la forma de «Estados competitivos» que priorizan el proceso de acumulación de capital en detrimento de la protección social de la población (citado por Dale, 1999). 
¿Hasta dónde influyen las agendas internacionales en los sistemas

educativos centroamericanos?

Un marco teórico para su análisis

Esta intencionalidad competitiva del Estado, en relación al proyecto educativo, puede analizarse a través de tres elementos que propone Dale (2000): a) mandato, b) capacidad y, c) gobernabilidad.

Por mandato, Dale (2000) entiende todo aquello que es deseable y legítimo que cumpla el sistema educativo; por ejemplo, ampliar la cobertura educativa y garantizar a su población el derecho a la educación sin discriminación alguna. Como capacidad, la capacidad efectiva de cumplir dicho mandato, esto es, la autonomía y los márgenes reales del Estado para conducir al sistema educativo hacia los objetivos establecidos. Es aquí donde afloran los problemas, ya que según Dale (2000), las políticas neoliberales empujan a los Estados hacia el recorte presupuestario y la ampliación de «mercados educativos», lo cual limita la capacidad de los Estados de cumplir con su mandato educativo. Y finalmente, la gobernabilidad, es decir, las formas de coordinación para alcanzar los objetivos que se conciben como deseables y factibles. Este punto es relevante, pues como explica Dale (1999), los Estados al estar cada vez más preocupados por apegarse a los marcos desarrollados por los organismos internacionales, los incluyen de forma directa o indirecta en el proceso de toma de decisiones sobre la educación nacional.

Al respecto Moutsios (2010) explica que la toma de decisiones sobre políticas educativas globales no se está dando en un espacio inter-nacional, ya que las políticas más influyentes no se formulan en un contexto dondelas fronteras y relaciones entre los Estados Nación están bien delimitadas. Tampoco en un espacio supra-nacional, pues las políticas educativas no se diseñan arriba ni más allá de los Estados, el proceso de toma de decisiones pasa de alguna forma por sus procesos burocráticos. Moutsios (2010), aboga por un espacio trans-nacional, instituido y sostenido por Estados Nación, organizaciones

Los articulos de la Revista Electrónica Paradigma del Instituto de Investigación y Evaluación Educativas y Sociales de la Universidad Pedagógica Nacional Francisco Morazán, de Honduras, se comparten bajo términos de la Licencia Creative Commons: Se permite que otros puedan descargar las obras y compartirlas con otras personas, siempre y cuando se reconozca su autoría, pero no se pueden cambiar de ninguna manera ni se pueden utilizar comercialmente. 
internacionales, entidades inter-estatales y corporaciones globales, donde las políticas y sus discursos atraviesan fronteras y, fluyen en y fuera del campo de poder de los Estados Nación.

No obstante, los Estados, miembros o no de estas organizaciones internacionales, no comparten el mismo nivel de influencia en el establecimiento de la agenda educativa global o nacional. Como expone Dale (1999), la naturaleza y el impacto de la globalización varía entre los países en función de su posición en la economía mundial. De acuerdo a las teorías del sistema-mundo capitalista y a las teorías latinoamericanas de la dependencia, la pauta de comparación entre los países es su nivel económico o de «progreso». En consecuencia, esta jerarquía global, expone Massey (2007), implica categorizar a los países en «desarrollados»y «subdesarrollados» de acuerdo a su nivel de «progreso».

Esto explicaría en parte la anuencia de los países periféricos de adaptarse a las agendas educativas internacionales para alcanzar el estándar de países desarrollados o bien, modernos. Como también la tendencia de los países del Centro a imponer sus agendas sobre los países periféricos. Esto nos conduce a la relación problemática entre organismos internacionales liderados generalmente por Estados de países «desarrollados» y sus agendas educativas, y sistemas educativos de países «subdesarrollados» como los centroamericanos.

Organismos internacionales y formación de politicas educativas nacionales.

De acuerdo a Dale y Robertson (2002), estudiar la influencia de la globalización sobre sistemas educativos nacionales, es igual a indagar sobre 
¿Hasta dónde influyen las agendas internacionales en los sistemas

educativos centroamericanos?

Un marco teórico para su análisis

las formas en que los organismos internacionales operan en territorios nacionales para transferir sus ideas. Pues los organismos internacionales, según Dale y Robertson (2002), constituyen los medios institucionales por excelencia, mediante los cuales, los valores del capitalismo global son trasladados a la esfera educativa nacional y global. Edwards $(2014,2018)$ llama a esta dinámica, «Procesos Internacionales de Formación de Políticas Educativas»-PIFPE-, es decir, «aquellos procesos que factores internacionales influencian y que son representativos nacionales del gobierno, de las autoridades educativas (...) y de los partidos políticos que se involucran para formular o autorizar el texto oficial que representa una política» (Edwards, 2014, p. 5).

Los PIFPE, de acuerdo a Edwards (2014), pueden caracterizarse por la atracción, la negociación, la imposición o la hibridación de políticas educativas internacionales. Dentro de estos procesos los organismos internacionales se valen de varias vías o «mecanismos» para trasladar sus ideas a los diferentes sistemas educativos nacionales. Estos «mecanismos» pueden ir de la imposición, armonización, diseminación, instalación de la interdependencia y la estandarización. La especificidad de cada uno se debe a la naturaleza de la relación entre los organismos internacionales y los sistemas educativos nacionales -de lo voluntario a la coacción-, los alcances de la política y su locus de viabilidad -externo, regional, nacional-, los actores que inician y forman parte del proceso -internacionales, nacionales-, la dimensión del poder -decisión consciente, intervención internacional directa-, y la naturaleza de los efectos de dicha influencia sobre la educación nacional.

En suma, el proceso de la globalización entendido como capitalismo global, ha alterado los procesos de reforma educativa nacionales, a través de la influencia de las agendas educativas internacionales promovidas por ciertos

Los articulos de la Revista Electrónica Paradigma del Instituto de Investigación y Evaluación Educativas y Sociales de la Universidad Pedagógica Nacional Francisco Morazán, de Honduras, se comparten bajo términos de la Licencia Creative Commons: Se permite que otros puedan descargar las obras y compartirlas con otras personas, siempre y cuando se reconozca su autoría, pero no se pueden cambiar de ninguna manera ni se pueden utilizar comercialmente. 
organismos internacionales. No obstante, los Estados-nación siguen ejerciendo una influencia importante en los procesos educativos nacionales, pero ¿cómo analizar este influjo?

Desde finales de los años ochenta y principios de los años noventa, el diseño y ejecución de PRE se abrió a otros actores nacionales como universidades, tanques de pensamiento, gremiales empresariales, sindicatos, etc. Esta nueva forma de estructuración de políticas educativas que conjuga a actores políticos y a actores sociales nacionales, indica Moutsios (2010), se concibió como una democratización de la toma de decisiones en materia educativa. No obstante, como vimos en el apartado anterior, si existen relaciones de poder entre oficiales de los organismos internacionales y de los sistemas educativos nacionales, lo mismo podemos esperar de las relaciones entre los actores nacionales con todo y su carga histórica en tanto clase social, género y etnia.

\section{Economía política crítica cultural: énfasis en el nivel nacional, la agencia y la hegemonía ideológica.}

Según Nederveen (2009), ningún Estado - Nación representa la unidad de «una» cultura nacional. Las naciones en su mayoría han sido formadas por conflictos de clase, etnia, religión y género. Por ende, Nederveen (2009) sostiene que los Estados - Nación constituyen ensamblajes conflictivos. Nuestra hipótesis es que estos ensamblajes conflictivos son reproducidos y a la vez resistidos por los actores nacionales que participaron en los PRE en cada país centroamericano. Y es que a estos actores se les asignó la responsabilidad de llegar a «consensos» sobre los nuevos proyectos educativos nacionales de posguerra a pesar de sus diferencias ideológicas. No obstante, partimos del supuesto que estos «consensos» ocultan conflictos de intereses y desiguales cuotas de influencia entre los actores. Para comprender este problema es 
necesario analizar el paradigma de la gobernanza educativa a la luz de la aproximación estratégica relacional de Jessop (2016), para luego ahondar en las fuerzas ideacionales que motivan las prácticas de los agentes, y el papel que juega la ideología y la hegemonía en las luchas de poder entre los mismos (Apple, 1993).

Gobernanza educativa desde la aproximación estratégica relacional.

Según Robertson y Dale (2013), el término education governance o gobernanza de la educación, ha sido acuñado para describir las actividades de gobierno relacionadas a la educación nacional que están siendo conducidas no solo por el gobierno (sistemas educativos nacionales), sino que también, por actores no gubernamentales. De acuerdo a Jessop (2008), el término «gobernanza» se refiere particularmente «a la autoorganización reflexiva de actores independientes involucrados en relaciones complejas de interdependencia recíproca», la cual, «se basa en el diálogo continuo y en el uso compartido de los recursos para desarrollar proyectos comunes» (2008, p. 60). Sin embargo, explica el mismo autor, «la gobernanza organizada sobre esta base no tiene por qué conducir a una simetría completa en las relaciones de poder ni a la igualdad absoluta en la distribución de beneficios» (2008, p. 60). Más bien, sigue Jessop $(2016,2008)$, esto resulta poco probable.

En América Latina, de acuerdo a Morales y Magalhaes (2013), la forma de diseñar e implementar políticas educativas ha transitado precisamente, de un modelo centrado en el "gobierno» a uno centrado en la "gobernanza», bajo la justificación que este último promueve una dinámica de toma de decisiones «más circular y compleja que consiste en diálogos, resolución de conflictos, construcción de alianzas y redes, estrategias y consensos entre

Los articulos de la Revista Electrónica Paradigma del Instituto de Investigación y Evaluación Educativas y Sociales de la Universidad Pedagógica Nacional Francisco Morazán, de Honduras, se comparten bajo términos de la Licencia Creative Commons: Se permite que otros puedan descargar las cbras y compartirlas con otras personas, siempre y cuando se reconozca su autoría, pero no se pueden cambiar de ninguna manera ni se pueden utilizar comercialmente. 
actores e intereses diversos» (Kjaer, 2010; citado por Morales y Magalhaes, 2013, p. 6).

Sin embargo, tanto Robertson y Dale (2013) como Verger et. al., (2016) arguyen que el modelo de la gobernanza al poner en juego distintos intereses, visiones sobre la educación y cuotas de poder de actores externos al gobierno tales como los organismos internacionales y el sector privado, debería alertarnos sobre posibles efectos negativos en los sistemas educativos nacionales, como lo son la privatización de la educación. Esto implica en última instancia, de acuerdo a Apple (1993) y Levin y Carnoy (1985), una lucha entre dos perspectivas de justicia social: derechos humanos básicos por una parte y los derechos de la propiedad privada y del consumidor por otra. Es decir, educación como bien público responsabilidad exclusiva del Estado, versus la apertura de mercados educativos de naturaleza privada.

Morales y Magalhaes (2013) basándose en Rhodes (1997) indican entonces, que las causas que motivaron la aplicación de los modelos de la gobernanza en la toma de decisiones educativas en Latinoamérica y en el globo, no deben buscarse en el impulso hacia la democracia, sino que en «las reformas del sector público inspiradas en las nuevas relaciones público-privadas y en el New Public Managment, originadas en los países anglosajones, y favorecidas por los programas de ajuste estructural» (2013, p. 6). Y es que la tendencia a reducir la centralidad del Estad y y su injerencia en aspectos económicos y sociales (políticas neoliberales), de acuerdo a Robertson y Dale (2013), se acompaña de la creación de mercados donde antes no existían, como por ejemplo, en la educación y la salud.

Explicado lo anterior, la gobernanza de la educación debería analizarse de acuerdo a las tres dimensiones que Robertson y Dale (2013) señalan: a) los 
¿Hasta dónde influyen las agendas internacionales en los sistemas

educativos centroamericanos?

Un marco teórico para su análisis

tipos de entidades y agentes con diferentes intereses y cuotas de poder que participan en los procesos de reforma educativa, $b$ ) las variadas plataformas o escalas de reglas y valores que se contraponen (internacionales, nacionales, locales) y, c) las actividades que los actores despliegan para influir en la toma de decisiones sobre lo educativo. Con estos elementos podríamos superar la concepción bastante noble o simple de la gobernanza como «consenso», para estudiar las relaciones de poder entre los actores involucrados en los PRE. Para ello será útil la aproximación estratégica relacional -SRA, en sus siglas en inglés- desarrollada por Jessop (2016), que tanto Edwards (2018) como Verger et al. (2016), aplicaron al estudio de las reformas educativas.

La SRA desarrollada por Jessop (2016) se basa en la producción de Nicos Poulantzas (1978, pp. 128-129; citado por Jessop, 2016, p. 54), quien fundamentado en el trabajo de Karl Marx y Antonio Gramsci, genera una teoría para comprender el carácter de clase del Estado y cómo éste, ha producido vías que privilegian a unos agentes e intereses sobre otros (Jessop, 2016, p. 54). Poulantzas propone que los conflictos y las contradicciones sociales amplias se reproducen dentro del espacio del Estado. En nuestro caso, podríamos decir lo mismo del sistema educativo nacional y de los PRE como parte del aparato estatal. Para comprender la SRA es importante esclarecer su concepción de «estructura» y «agencia», y la interdependencia entre ambas.

La «estructura», según la SRA, es una composición de oportunidades y restricciones que se activan dependiendo del tipo de agente (Jessop, 2016). Mientras que, explica Jessop (2016), la «agencia» depende de las capacidades estratégicas diferenciales que ejercen actores particulares, posicionados de forma desigual en diversas estructuras sociales. Las estructuras entonces, restringen o privilegian la capacidad de agencia de los individuos y colectividades, de acuerdo al tipo de agente que representan en términos de

Los articulos de la Revista Electrónica Paradigma del Instituto de Investigación y Evaluación Educativas y Sociales de la Universidad Pedagógica Nacional Francisco Morazán, de Honduras, se comparten bajo términos de la Licencia Creative Commons: Se permite que otros puedan descargar las obras y compartirlas con otras personas, siempre y cuando se reconozca su autoría, pero no se pueden cambiar de ninguna manera ni se pueden utilizar comercialmente. 
clase, etnia, religión y género, es decir, de sus recursos y posición social. Por lo que las estructuras sociales son estratégicamente selectivas, más que absolutamente restrictivas (Jessop, 2018, p. 55).

En el caso de los procesos de reforma educativa en Centroamérica, estos se llevaron a cabo al menos en apariencia, con la participación de diversos sectores sociales más allá de los oficiales del Estado. Estos actores representaron a variadas instituciones y organizaciones como las gremiales empresariales, sindicatos de maestros, organizaciones indígenas y de mujeres, nuevos partidos políticos de izquierda (formados por ex guerrilla), universidades públicas y privadas, think tanks, y cómo hemos venido desarrollando, oficiales de organismos internacionales, entre otros. Estos actores sociales y políticos, bajo el modelo de la gobernanza, fueron convocados por sistemas educativos nacionales para participar en la toma de decisiones sobre el contenido y fines de los PRE. Estos actores sociales y políticos se encontraban constreñidos o habilitados de forma diferencial en función de su posición social (clase, género, religión y etnia), por la «estructura» que los convocó, es decir, sistemas educativos nacionales que forman parte de Estados centroamericanos particulares. Los sistemas educativos nacionales suponemos, privilegiaron los intereses de unos actores sobre otros, limitando o fortaleciendo la capacidad de influencia de unos actores y sus visiones.

Las oportunidades que las «estructuras» abren a cierto tipo de actores, de acuerdo a Danemark, Ekstriöm, Kakobsen, \& Karlsson, (2016), depende de la congruencia de intereses entre estos actores y la «estructura», es decir, los sistemas educativos nacionales. Jessop (2018) subraya así, el poder transformativo o reproductivo de las acciones de los agentes sobre las estructuras. Es decir, reproducir el balance de fuerzas más amplio en 
¿Hasta dónde influyen las agendas internacionales en los sistemas

educativos centroamericanos?

Un marco teórico para su análisis

estructuras particulares como los sistemas educativos o bien, intentar transformarlo a través de acciones estratégicas. Como insiste Danemark et. al., «la fuerza que en primer lugar debemos tomar en consideración es la intencionalidad de los agentes, es decir, la capacidad de formular proyectos (metas) y establecer estrategias de acción para alcanzarlos» (2016, p. 136). De acuerdo a Edwards (2018) basándose en Jessop (2001), los actores interpretan el contexto político e institucional en el que se encuentran inmersos y actúan estratégicamente para lograr sus objetivos dentro de restricciones y oportunidades selectivas, fundamentándose en sus propias experiencias e intuiciones políticas. Los actores por lo general saben qué acciones son premiadas en el contexto en el que se desenvuelven, indica Edwards, (2018), pero a su vez, nunca están totalmente conscientes de las condicionantes que afectan sus acciones, así que no hay garantías que logren sus objetivos (Jessop, 2016). Esto nos introduce en el terreno de la subjetividad de los actores involucrados en los PRE.

De acuerdo a Verger et. al., (2016), releyendo a Jessop (2010), dentro del marco de la EPCC el dominio ideacional -cultura, normas, ideas, aprendizaje, semiosis- se encuentra incrustado en los dominios económicos y políticos, y como tal, no puede analizarse como una dimensión separada. Las ideas y acciones de los actores se encuentran condicionadas por fuerzas económicas y políticas (estructura social), pero a su vez, las fuerzas económicas y políticas no podrían existir sin las acciones de los agentes. Como Danemark et al., explican: «(...) las estructuras sociales y sus prácticas, posiciones y reglas asociadas, deben existir para que sean posibles las acciones de las personas, de la misma manera que las acciones deben existir para que las estructuras sociales sean posibles» (2016, p. 115). Aplicando esto a nuestra investigación, las fuerzas económicas y políticas que influyeron los PRE en Centroamérica

Los articulos de la Revista Electrónica Paradigma del Instituto de Investigación y Evaluación Educativas y Sociales de la Universidad Pedagógica Nacional Francisco Morazán, de Honduras, se comparten bajo términos de la Licencia Creative reconozca su autoría, pero no se pueden cambiar de ninguna manera ni se pueden utilizar comercialmente. 
condicionaron diferencialmente las capacidades de acción de los diversos actores convocados, pero los PRE no pudieron ser posibles sin la acción de actores concretos. Ahondemos a continuación en las fuerzas ideacionales que motivaron las prácticas de los agentes nacionales en los PRE.

Fuerzas de tipo ideacional: paradigmas de políticas educativas y hegemonía ideológica.

Un paradigma político o policy paradigm, según Verger et al., (2016), constituye una «estructura» de tipo ideacional que discrimina y selecciona los «discursos aceptados» y «buenas prácticas» relacionadas a las políticas educativas globales. Estos paradigmas políticos fungen como marcos de referencia a través de los cuales los actores que participan en los PRE o policymakers, interpretan su realidad y toman decisiones basados en un conjunto estándar de ideas sobre qué son las políticas educativas, cuál es la naturaleza de los problemas educativos, el tipo de instrumentos que pueden utilizarse para solucionarlos y los objetivos que las reformas educativas deberían perseguir. Según Verger et al. (2016) y Moustios (2010), el rol que los organismos internacionales han adquirido como «expertos educativos» es vital para entender la configuración y reproducción de estos paradigmas.

La formación de políticas educativas se tiende a ver como un proceso fundamentado en evidencias explica Moustios (2010), y en la actualidad el rol de generar «conocimiento científico fiable» sobre políticas educativas se ha relegado a los organismos internacionales. Éstos han adoptado un rol de autoridad mundial en el tema educativo en dos sentidos: a) son una autoridad pues manejan datos y demuestran expertise, y b) están en la autoridad de ejercer poder sobre los sistemas educativos nacionales pues éstos reconocen 
¿Hasta dónde influyen las agendas internacionales en los sistemas

educativos centroamericanos?

Un marco teórico para su análisis

su legitimidad como expertos. Todo ello predispone, de acuerdo a Verger, et. al., (2016), a que los policymakers nacionales acepten los servicios de expertos de organismos internacionales y estén más receptivos ante su opinión sobre la manera de reformar los sistemas educativos nacionales. La diseminación de los «paradigmas internacionales sobre las políticas educativas», no se deben a su efectividad, rigor científico (Verger, et. al., 2016, Moustios, 2010) o a que impulsan la modernización de los países per se (Dale, 2000), sino que a las acciones de promoción y persuasión de estos expertos internacionales para enmarcar las percepciones de los policymakers según ciertos paradigmas políticos.

El proceso clave aquí es la semiosis, es decir, formas de producción social de significados que influyen sobre la decisión de los individuos (Verger, et. al, 2016). De acuerdo a Verger et al., (2016), las semiosis tiene una dimensión intersubjetiva con el potencial de producir estructuras ideacionales o bien, «paradigmas de políticas educativas», con poderes causales sobre las preferencias individuales y decisiones de los policymakers (2016, p. 26). En este sentido, Moustios (2010) argumenta que el rol de los expertos es brindar soluciones «encajonadas» a problemas educativos creados, diseminar dichos «paquetes» de ideas a las naciones y persuadir a policymakers nacionales a que las apliquen.

No obstante, surge una pregunta: ¿por qué policymakers de países diversos adoptan y aplican dichos paradigmas políticos no importando las diferencias culturales e históricas de cada país? Consideramos que los estudios de Apple (1993) sobre las políticas del conocimiento oficial pueden ayudarnos comprender estos procesos subjetivos y sociales de adopción de paradigmas políticos en tanto hegemonía ideológica.

Los articulos de la Revista Electrónica Paradigma del Instituto de Investigación y Evaluación Educativas y Sociales de la Universidad Pedagógica Nacional Francisco Morazán, de Honduras, se comparten bajo términos de la Licencia Creative Commons: Se permite que otros puedan descargar las cbras y compartirlas con otras personas, siempre y
reconozca su autoría, pero no se pueden cambiar de ninguna manera ni se pueden utilizar comercialmente. 
Según Apple (1993), la decisión de legitimar el conocimiento de unos grupos como el oficial, y deslegitimar el conocimiento de otros grupos sociales, dice algo sobre quien tiene el poder en una sociedad particular. Las luchas que vemos entre actores políticos y sociales, del sector privado y el sector público, de organismos internacionales y de entidades nacionales, por influir en la organización del contenido de las reformas a la educación y los planes educativos que las echarán adelante, en el marco de la nueva gobernanza educativa, no son otra cosa, que pugnas políticas por legitimar la visión de mundo de unos grupos sobre otros, es decir, como veremos, pugnas por la hegemonía ideológica a través de la educación.

Apple (1993), enmarca este proceso en lo que llama «política del conocimiento oficial». Y es que unos grupos interpretarán el conocimiento oficial como una descripción neutral del mundo que debe ser enseñada e institucionalizada a través del sistema educativo nacional; mientras que para otros grupos, el conocimiento oficial representa una visión elitista del mundo y dela educación que beneficia a ciertos grupos sociales. Esta visión del mundo oficial, tiene que ver con relaciones de poder en tanto clase, etnia, religión y género de cada país. Por lo que las políticas del conocimiento oficial nacionales no pueden remitirse exclusivamente y en abstracto a la perspectiva de los organismos internacionales, tienen raíces nacionales. Tres constructos resultan centrales para comprender esto: ideología, hegemonía, y tradición selectiva.

En el texto Thinking 'right' in the USA: ideological transformations in an age of conservatism, Michael Apple (1993b), argumenta que para entender el término «ideología», no debemos empezar peguntándonos qué es lo falso en ella. Más bien, tendríamos que preguntarnos qué es verdad en ella, y cuáles son sus conexiones con la experiencia vivida de las personas. Esto fisura la 
¿Hasta dónde influyen las agendas internacionales en los sistemas

educativos centroamericanos?

Un marco teórico para su análisis

concepción marxista estructuralista de la ideología (Althusser, 1988) como una falsa conciencia que es determinada por la estructura económica e impuesta a individuos que la reproducen inconscientemente y sin mayor capacidad de agencia. Pues las ideologías para ser efectivas, explica Apple (1993), deben conectarse a problemas y experiencias reales de la gente y, es a través de este proceso, que una visión de mundo se configura históricamente como la hegemónica en una sociedad dada. Es decir, cuando ciertos sectores de la sociedad logran conectar sus programas económicos, políticos y educativos, a deseos y miedos de la población y reorganizar sentimientos populares.

Y es que para Gramsci (1971), -base teórica de Apple-, a diferencia de Marx, la sociedad no se encuentra determinada exclusivamente por las relaciones económicas (estructura), sino que la superestructura (cultura) juega un papel elemental para preservar la hegemonía de la clase dominante (Miranda, 2000, p.17). Pues el dominio del poder político de las clases dirigentes está asentado no sólo en el uso de la fuerza, sino que también en la capacidad de generar consensos entre diversos grupos sociales (recordemos el proceso de la gobernanza de la educación). La clase dirigente, explica Gramsci (1971), más que imponer la ideología de las clases altas a la población, debe organizarse y luchar por establecer una conciencia colectiva acorde sus objetivos políticos, por medio de un liderazgo moral e intelectual sobre las clases «aliadas». Estas tensiones y alianzas, que en la actualidad se dan en el marco de la gobernanza, se concretan en luchas dentro del campo político del Estado, donde los sistemas educativos nacionales se encuentran. En otras palabras, toda ideología para llegar a ser hegemónica, debe incluir y distorsionar anhelos populares (alimento, vivienda, trabajo, salud, educación, etc.) con el propósito de obtener el consentimiento de las clases subalternas y con ello, hegemonizar una visión de mundo supuestamente incluyente.

<Paradigma> Revista de Investigación Educativa 40 Noviembre 2018/ ISSN: 1817-4221

Los articulos de la Revista Electrónica Paradigma del Instituto de Investigación y Evaluación Educativas y Sociales de la Universidad Pedagógica Nacional Francisco Morazán, de Honduras, se comparten bajo términos de la Licencia Creative Commons: Se permite que otros puedan descargar las obras y compartirlas con otras personas, siempre y cuando se reconozca su autoría, pero no se pueden cambiar de ninguna manera ni se pueden utilizar comercialmente. 
En el caso de los Estados Unidos de los años noventa, Apple $(1993,1993 b)$ descubrió que la reformas educativas que se impulsaron en la época, cobraron sentido con el regreso de una derecha neoconservadora que supo articularse a la clase corporativa y sus valores neoliberales. Ambas ideologías a pesar de sus aparentes diferencias coalicionaron en lo que Dale llamó una «modernización conservadora»(Apple, 1993b), que «libera» los fines y deseos económicos de los individuos mientras «controla» sus propósitos sociales. Apple $(1993,1993 b)$ nombró esta corriente política como «populismo autoritario», e incluyó las políticas de Reagan en los Estados Unidos y de Tatcher en Inglaterra. Bajo este contexto político Apple (1993) se preguntó cómo la población estadounidense aceptó el cambio de políticas educativas que promovían el derecho público a la educación (Estado keynesiano), por políticas educativas neoliberales que privilegiaron la competencia y el desarrollo de mercados educativos financiados en su mayoría por los bolsillos de la misma población. En otras palabras, cómo el discurso neoliberal de la reforma educativa conectó con los deseos y miedos de la población para que fuera aceptado y naturalizado.

De acuerdo a Apple (1993), los movimientos feministas, de diversidad sexual, de estudiantes y trabajadores y en especial, el «black power» durante los sesentas diseminaron nuevos significados culturales sobre la identidad individual, familia y comunidad en el tejido social estadounidense, trastocando las relaciones sociales cotidianas. En lo educativo esto se reflejó en la expansión de programas bilingües y el acceso de mujeres negras, hispanas e indígenas americanas a escuelas secundarias y universidades (Apple 1993). Y el éxito de las políticas neoliberales que la administración de Reagan promovió y que Bush continuó, explica Apple (1993) se deben a los miedos que estas libertades ganadas por las minorías étnicas desataron en la clase media blanca tradicional de los Estados Unidos. 
¿Hasta dónde influyen las agendas internacionales en los sistemas

educativos centroamericanos?

Un marco teórico para su análisis

El punto de conexión entre los sentimientos populares y los programas populistas autoritarios, fue un sentido de pérdida de control sobre el conocimiento y los valores que debían ser transmitidos a las nuevas generaciones en las escuelas y familias, como también un sentido de inseguridad económica y personal por la inclusión de las minorías étnicas en espacios escolares y laborales tradicionalmente destinados para la clase media blanca (Apple, 1993b, p. 57). Esto motivó el apoyo de la población ante reformas educativas encaminadas a la formación de un currículo nacional que estandarizara y controlara los contenidos educativos a impartir (Apple, 1993), y a la configuración de un sistema educativo «meritocrático» que selecciona a sus integrantes con base en méritos individuales, mientras invisibiliza cuestiones como el género, raza y clase en el fracaso escolar y en la obtención de privilegios. Esto naturalizó la promoción de un mercado educativo basado en la competencia y en estándares educativos aparentemente neutrales, a la vez que estimulaba la representación de los pobres y las minorías étnicas como «delincuentes y haraganes» que sólo buscan beneficios gratuitos del Estado.

Así, la nueva derecha creó una nueva síntesis ideológica retomando aspectos culturales del pasado para promover un nuevo acuerdo hegemónico que combinara los intereses de las élites políticas y económicas, y los miedos de una clase media y trabajadora preocupada por la seguridad, la familia, la movilidad económica y la trasmisión del conocimiento y los valores tradicionales en la escuela y la familia. A este proceso, Apple lo llama «tradición selectiva», es decir, «el modo en que, de todo un área posible del pasado y presente, se elige poner el énfasis en determinados significados y prácticas, mientras se desestiman y excluyen otros» (1979, p. 17). 
De acuerdo a Apple (1993b) este fue el trasfondo político, económico y cultural que motivó reformas educativas en los Estados Unidos y que procuró entre otras cosas, que la mayoría de maestros creyeran y transmitieran dichos valores en sus escuelas, fungiendo como «intelectuales orgánicos» que ayudan a legitimar en los sistemas educativos nacionales ciertos discursos ideológicos, haciendo de los mismos, conceptos aparentemente cotidianos y neutrales (Apple, 1979).

\section{Conclusiones}

A manera de hipótesis

Los marcos teóricos no son estáticos, ni mucho menos verdades absolutas. Son herramientas analíticas sensibles a modificaciones al ser interpelados por el fenómeno social que desea estudiarse, evaluando así su utilidad. Decimos esto porque concluiremos este ensayo con una serie de presupuestos y recomendaciones que deberán probarse a través de investigaciones sobre las reformas a la educación en Centroamérica.

Habiendo dicho lo anterior, suponemos que la visión de mundo que se hegemoniza a través de los PRE no es el resultado mecánico de organismos internacionales que «imponen» agendas educativas globales acordes a los valores del capitalismo global, sobre sistemas educativos nacionales pasivos que integran, en el marco de la gobernanza, a actores diversos sin capacidad de agencia. Comprender la influencia de los paradigmas de políticas educativas internacionales sobre los PRE pasa por entender las pugnas político-ideológicas entre actores sociales y políticos nacionales, que toman sentido en un contexto histórico y cultural determinado. 
¿Hasta dónde influyen las agendas internacionales en los sistemas

educativos centroamericanos?

Un marco teórico para su análisis

En el caso centroamericano ameritará estudiar a fondo la transición de Estados autoritarios a Estados democráticos, durante finales de los años ochenta y principios de los años noventa (Torres, 2007), para entender las herencias que la guerra pudo dejar en las relaciones sociales entre los actores y en la ideología de la educación que intentó instalarse a través de la sociedad y ciudadano posguerra que deseaba formarse; como también las esperanzas y nuevas voces que el período democrático abrió e incluyó en ese momento insigne de la historia de la región. Asimismo, estudiar la influencia de la crisis económica que dejó la guerra y la necesidad de los países centroamericanos de insertarse a la nueva economía mundial, lo cual demandó de los sistemas educativos la formación de un nuevo tipo de trabajador y consumidor.

Tomando en cuenta que a pesar de sus similitudes, los países centroamericanos ameritan estudiarse desde sus particularidades, recomendamos reflexionar sobre los siguientes puntos:

a) Los organismos internacionales involucrados en el PRE del país en cuestión, sus discursos educativos y las formas o «mecanismos» que utilizaron para diseminar sus ideas en los sistemas educativos nacionales y con qué actores nacionales tendían a aliarse.

b) Qué actores nacionales son los que se «identificaron» con el discurso educativo internacional (¿elites económicas, organizaciones indígenas y think tanks etc.?), y tratar de entender, según su posición en la jerarquía social, por qué estos actores nacionales se «conectaron» con dicha narrativa, qué de su visión de mundo y experiencias los hace propensos a adoptar el discurso de los organismos internacionales y qué beneficios obtienen al hacerlo.

c) Estudiar si estos «actores afines» reproducen en discurso y en la práctica las políticas educativas internacionales tal cual, o bien, las adaptan y resignifican según el contexto cultural nacional, con lo cual, lograrían posiblemente que otros actores nacionales se identifiquen.

Los articulos de la Revista Electrónica Paradigma del Instituto de Investigación y Evaluación Educativas y Sociales de la Universidad Pedagógica Nacional Francisco Morazán, de Honduras, se comparten bajo términos de la Licencia Creative Commons: Se permite que otros puedan descargar las obras y compartirlas con otras personas, siempre y
reconozca su autoría, pero no se pueden cambiar de ninguna manera ni se pueden utilizar comercialmente. 
d) Estudiar las alianzas y desavenencias que se forman entre los actores en los PRE, en el marco de la gobernanza educativa, tomando en cuenta sus congruencias y diferencias en tanto clase social, etnia, género y religión, y su posición en cuanto a las agendas educativas internacionales;

e) Explorar los contenidos de estos «consensos» para la reforma educativa y preguntarse qué contenidos de las agendas educativas internacionales y qué imaginarios y discursos del bagaje histórico y cultural del país fueron o no seleccionados y cuáles fueron sus efectos en la población.

f) Analizar en la práctica qué del discurso de las reformas educativas fue realmente aplicado, y cómo y a qué sectores sociales nacionales e internacionales benefició.

\section{Bibliografía}

Apple, M. (1979). Ideología y currículo. Routledge \& Kegan Paul. Ediciones Akal, S.A., 1986, 2008, para la lengua española, traducción de Rafael Lassaletta

Apple, M. (1993). The politics of official knowledge: Does a national curriculum make sense? Teachers College Record, 95 (2), 222-241. Por Teachers College, Columbia University

Apple, M. (1993b). Thinking 'Right' in the USA: Ideological transformations in an age of conservatism. In Schooling Reform in hard times (pp. 4962). London \& Washington, D. C.: The Falmer Press, member of Taylor \& Francis Group

Bonal, X. (1998). Sociología de la educación: una aproximación crítica a las corrientes contemporáneas. Barcelona: Ediciones Paidós Ibérica, S.A.

Dale, R. (1999). Specifying globalization effects on national policy: A focus on the mechanisms. Journal of Education Policy, 141, 1-17 
¿Hasta dónde influyen las agendas internacionales en los sistemas

educativos centroamericanos?

Un marco teórico para su análisis

Dale, R. (2000). Globalization and education: Demonstrating a 'common world educational culture' or locating a 'globally structured educational agenda'? Educational Theory, 50(4), 427-448

Danemark, B., Ekstriöm, M., Kakobsen, L., \& Karlsson, J. (2016). Estructuras sociales y acción humana. In Explicando la sociedad, el realismo crítico en las ciencias sociales (pp. 113-150). El Salvador: UCA Editores. Traducción, Mario Zetino Duarte

Edwards, B. (2014). ¿Cómo analizar la influencia de los actores e ideas internacionales en la formación de políticas educativas nacionales? Una propuesta de un marco de análisis y su aplicación a un caso de El Salvador. Archivos analíticos de políticas educativa, 22(12)

Edwards, B. (2018). Critical international political economy and mechanisms/ pathways of influence (Unpublished PhD. thesis). University of Hawaii, Department of Educational Foundations. Capítulo 3, tesis doctoral. Author's Proof

Gramsci, A. (1971). Selections from the prison notebooks. London: Lawrence \& Wishart.

Edites and translated by Quentin Hoare and Geoffrey Nowell Smith, ElecBook, London 1999.

Jessop, B. (2016). The state as a social relation. In The State, past, present, future (1st ed., pp. 53-90). Malden, USA, \& Cambridge, UK: Polity Press

Jessop, R. (2008). Capitalismo y tipo de estado capitalista. In El futuro del estado capitalista. Madrid: Los libros de la catarata.

Massey, D. (2006). Space, time and political responsibility in the midst of global inequality. Erdkunde, Germany,89-95

Los articulos de la Revista Electrónica Paradigma del Instituto de Investigación y Evaluación Educativas y Sociales de la Universidad Pedagógica Nacional Francisco Morazán, de Honduras, se comparten bajo términos de la Licencia Creative reconozca su autoría, pero no se pueden cambiar de ninguna manera ni se pueden utilizar comercialmente. 
Miranda, G. (2006). Gramsci y el proceso hegemónico educativo. Revista Electrónica Educare, Universidad Nacional; Heredia, Costa Rica, IX (2), 13-39

Morales Ulloa, R., \& Magalhaes, A. (2013). Visiones, tensiones y resultados. La nueva gobernanza de la educación en Honduras. Education Policy Analysis Archives/Archivos Analíticos De Politicas Educativas, 21, 120. Arizona State University, Arizona, Estados Unidos

Moutsios, S. (2010). Power, politics and transnational policy-making in education. Globalization, Societies and Education, 8(1), 121-141. Publisher Routledge

Naderveen Pieterse, J. (2009). Globalization as hybridization. In Globalization and culture (pp. 45-65).

Robertson, S., \& Dale, R. (2013). The social justice implications of privatization in education governance frameworks: A relational account. Oxford Review of Education, 39(4), 426-445. Publisher Routledge

Robinson, W. (2007). Theories of globalization. In The blackwell companion to globalization (pp. 125-143). Australia: Blackwell Publishing

Tarabini, A., \& Bonal, X. (2011). Globalización y política educativa: los mecanismos como método. Revista de Educación, 235-255. Retrieved 2011

Torres Rivas, E. (2007). La piel de Centroamérica, una visión epidérmica de setenta y cinco años de su historia (1st ed.). San José, Costa Rica: Flacso.

Verger, A., Fontdevila, C., \& Zancajo, A. (2016). The political economy of global education reform. In The privatization of education, a political economy of global education reform (pp. 15-34). New York: Teachers college press 